\title{
Unequal Error Protection Based on Objective Video Evaluation Model
}

\author{
Wen Ji \\ Institute of Computing \\ Technology, Chinese \\ Academy of Sciences \\ Beijing, China \\ jiwen@ict.ac.cn
}

\author{
Yiqiang Chen \\ Institute of Computing \\ Technology, Chinese \\ Academy of Sciences \\ Beijing, China \\ yqchen@ict.ac.cn
}

\author{
Min Chen \\ Dept. of Electrical and Spreadtrum Communication \\ Computer Engineering, Univ. Co., Ltd. \\ of British Columbia, Shanghai, China \\ BC, Canada \\ minchen@ece.ubc.ca Yi.Kang@spreadtrum.com
}

\begin{abstract}
Usually unequal error protection schemes mostly focus on protecting video data parameters with unequal rates or levels depending on their sensitivities to errors. This paper first proposes a novel effective and reliable objective video evaluation model based on fuzzy synthetic judgment. This model highlights a comprehensive evaluation by taking account of multiple properties of the compressed video which affect the whole video sequence, such as quality, fluency and motion information. Secondly, a novel unequal protection scheme is proposed. The protection levels are calculated according to the output of the fuzzy evaluation results. The whole coded video can be transmitted efficiently since the protection is based on the video quality evaluation. Simulation results demonstrate the advantages of the fuzzy objective video quality evaluation model, especially in comprehensive judgment of the whole video, and show the subjective quality improvement obtained by applying the proposed unequal protection approach.
\end{abstract}

\section{Keywords}

Unequal error protection(UEP), video quality evaluation, fuzzy theory.

\section{INTRODUCTION}

In video communication system, since the parameters of video compression algorithms present different sensitivities to errors and different contributions to overall decoded quality, proper unequal protection methods have been proven to be very promising to resolve this problem. But these methods concentrate on protecting video data corresponding to their sensitivity to errors and their contribution to overall video quality. For instance, unequal error protection method provides different or proportional level of protection, according to different frame types and partitioned data [1][2]. These are mainly summarized for considering the error sensitivity and propagation of frame data. So do multi-layer video streams. In this paper, we take the video characteristics into account sufficiently, and give a judgment method of the coded video frames so as to provide best unequal

Permission to make digital or hard copies of all or part of this work for personal or classroom use is granted without fee provided that copies are not made or distributed for profit or commercial advantage and that copies bear this notice and the full citation on the first page. To copy otherwise, or republish, to post on servers or to redistribute to lists, requires prior specific permission and/or a fee.

Mobimedia'07, Month 8, 2007, Nafpaktos, Aitolokarnania, Greece

Copyright 2007 ICST 978-963-06-2670-5 protection combined with multiple properties of the compressed video which affect the whole video sequence. First, we give a new effective and reliable objective video evaluation model based on fuzzy synthetic judgment [7] and describe systemically the essential design of the model. Coded video features such as quality, fluency, motion info, frame type and the interval are enrolled in the model as affecting factors. The overall evaluation results of coded frames can be derived from the model, which consist of the weight of protection and error correction capabilities so as to make communication more efficiently.

\section{PROPOSED ALGORITHM}

\subsection{Video Evaluation Model}

Before explaining our design criteria, we build a video evaluation model based on fuzzy synthetic judgment [3], and give the details as follows. Let $U=\left\{u_{1}, u_{2}, \ldots, u_{\mathrm{n}}\right\}$ be the set of video quality affecting factors; Let evaluation results' set be $V=\left\{v_{1}, v_{2}, \ldots, v_{\mathrm{m}}\right\}$; Let $R=\left(r_{i j}\right)_{n \times m}, 0<r_{i j}<1$ be a fuzzy relation from $U$ to $V$. If a fuzzy subset $A=\forall U$ and fuzzy subset $B=\forall V$ meet $B=A \circ R$, there is a fuzzy evaluation from fuzzy set $U$ to fuzzy set $V$. According to extension theorem, given $R \in \mathcal{F}(U \times V)$, a mapping $T_{R}: F(U) \rightarrow F(V)$, which meet $A \rightarrow T_{R}(A)=A \circ R$, the fuzzy evaluation is unique. Therefore, finding an objective compressed video quality evaluation model can be equivalent to solve a fuzzy synthetic evaluation problem. Obviously, the final evaluation results have close relationship with the building of single- and multi- affecting factors. Video quality affecting factors' set includes $l$ subsets of $U_{i},(i=1,2, \ldots, l)$, which satisfies $\bigcup_{i=1}^{l} U_{i}=$ $U$. Thus, according to the fuzzy model, evaluation vectors corresponding to each factors constitute the evaluation matrix $R=\left(B_{1}, B_{2}, \ldots, B_{l}\right)^{T}=\left(\begin{array}{ccc}b_{11} & \cdots & b_{1 m} \\ \vdots & \ddots & \vdots \\ b_{l 1} & \cdots & b_{l m}\end{array}\right)$.The weights vector of single- factor is $A=\left(a_{1}, a_{2}, \ldots, a_{l}\right)$, where $i=1,2, \ldots, l$. Consequently, the results of the fuzzy synthetic evaluation can be written as follows: $B=A \circ R=\left(a_{1}, a_{2}, \ldots, a_{l}\right) \circ\left(\begin{array}{ccc}b_{11} & \ldots & b_{1 m} \\ \vdots & \ddots & \vdots \\ b_{l 1} & \cdots & b_{l m}\end{array}\right)=$ $\left(\sum_{i=1}^{l} a_{1} b_{i 1}, \sum_{i=1}^{l} a_{2} b_{i 2}, \ldots, \sum_{i=1}^{l} a_{l} b_{i m}\right)$. 
In order to make our proposed model have well compatibility with other video quality evaluation models or standards, model extension is provided by introducing corresponding multiaffecting factors into the fuzzy video evaluation model. For instance, most of the other evaluation methods can be annexed to the fuzzy systematic video evaluation model as multi-affecting factors, such as multi-channel properties of the HVS, perceptual distortion assessment, 3D-WSNR, automatic video quality assessment on the metric, perceptual prefiltering model [4-6]. According to inductive inference theorem, the reliability of the fuzzy systematic video evaluation model will be enhanced when the relation of these models belongs to implication.

For building multi- affecting factors, the weights vector is written as $A_{j}=\left(a_{1}, a_{2}, \ldots, a_{l}\right)$ according to each $U_{j}$, the evaluation results are deduced from the second fuzzy transform, described as $B=A_{j} \circ R$. If $A_{j}$ is still a subset, we can get the final evaluation result $B=A \circ R$ by a recursion method. Therefore, the evaluation model has well capability of compatibility.

\subsection{Optimizing Model to UEP transmission system}

For an efficient transmission of compressed video, the possibility of considering different code rates for the protection of different frames is proposed: different frames are protected according to their different evaluation results using above video evaluation model based on fuzzy synthetic judgment. In this scheme, video features including quality, motion information, interval and sensitivity are involved to build the unequal protection. The weight of protecting video frames in unequal proportions can be derived from the model. The communication becomes more efficient due to combining video quality evaluation with the transmission model.

The fuzzy evaluation model presents a new evaluation method with emphasis on the systematization, addressing for video characters including video quality, fluency and motion information. Therefore, $U=\left\{u_{1}, u_{2}, u_{3}, u_{4}, u_{5}\right\}$ is specified as the set of video quality affecting factors, where $u_{1}$ is MSE, $u_{2}$ is the interval between adjacent frames, $u_{3}$ represents motion characters, $u_{4}$ means the quantization parameter, and $u_{5}$ is the frame type in a GOP (Group of Pictures). Consequently, the factor vectors of the fuzzy synthetic evaluation model can be taken as below.

$R_{1}=\left\{M S E_{1}, \cdots, M S E_{n}\right\}$ : the mean-squared-error of each encoded frame. This judgment vector is complied with the conventional video quality measurement.

$R_{2}=\left\{\Delta t_{1}, \cdots, \Delta t_{n}\right\}$ : the intervals of adjacent frames. The objective PSNR or MSE results do not always provide a reliable metric for the continuity performance evaluation of a video sequence. Since video signals are three-dimensional, the temporal factor plays a major role in the overall assessment, especially in the aspects of wobble and fluency.

$R_{3}=\left\{\right.$ motion $_{1}, \cdots$, motion $\left._{n}\right\}$ : the motion vectors based on the motion estimation in each block. The main limitation of PSNR or MSE is that they do not correlate well with subjective quality evaluations. For a fair evaluation of a compressed video sequence, motion information should also be included. The sequences have a strong relativity with one or several preceding images referred. Different prediction modes affect the quality of coded video. Therefore, motion estimation information is taken into account as a factor in this model.

$R_{4}=\left\{\sigma_{1}, \cdots \sigma_{n}\right\}:$ the parameters set of quantization. The compression process has a close relationship with the quantization of the transformed coefficients. The quantizer is regarded as the most important component of the video encoder since it controls both the coding efficiency and the quality of reconstructed video sequence. Most video compressing algorithms include many techniques such as flow control, optimal search of motion, etc. which can be adapted simply by these coefficients. The finial quality of compressed video is closely related to the quantization, thus, the reliability of evaluation could be improved when the quantization step size is involved in factor vectors.

$R_{5}=\left\{c_{1}, \cdots c_{n}\right\}$ : the set of frame type. Generally, frame can be divided into three types (i.e., intra, inter predicted and bidirectional frame) in a GOP.

The above five video quality affecting factors are all within 'or' or 'implication' relations each other. According to inductive inference theorem, the reliability of synthetic evaluation results can be enhanced. The variability range of factors value except $R_{1}$ is in inverse proportion to the value of judgment result, which is consistent with the tendency of MSE .

As some extreme examples, when weight assignment vector of affecting factor becomes $A=(1,0,0,0,0)$, the video quality evaluation degenerates to PSNR measurement system which being widely used. When $A=(0,1,0,0,0)$ or $(0,0,1,0,0)$, it degenerates to judgment of fluency and wobble of video sequence. As a result, many evaluations in research can be included to the subset of the fuzzy video quality evaluation model.

Due to the limited resource in wireless communication, delivery adequate perceptual quality of video to users is attaching more importance. Previous works on unequal error protection are based mainly on the sensitivity of compressed data. In this paper, the compressed video quality evaluation model provides a perceptual mode decision method which determines different video protection segment levels. The proposed UEP scheme is depicted in Fig 1. The values of each element in fuzzy judgment results compose unequal protection weights, so that the perceptual features of compressed video are optimized resulting from the error control paradigm or other bit rates protected allocation scheme. Therefore, the weighting decision is come out from the output of the fuzzy evaluation results. Evaluation results vectors corresponding to each factor compose the index matrix, which is attributed to the steps according to UEP states. Furthermore, a threshold is provided to adjust the effect degree from the evaluation model. Thus, the whole video sequence is transmitted efficiently since the protection level is based on the video quality evaluation. Given the frame based protection as example, according to each frame in the GOP, the protection is calculated exactly so that the final quality of the overall reconstructed video reaches its maximum.

Furthermore, we provide an extension interface in this method. For instance, it can include the feedback from the following 
control modules. The application involves error resilience or video transcoding, etc. Besides these, the extension is not limited to feedback but the forward rate allocation such as the rate allocation in source coding.

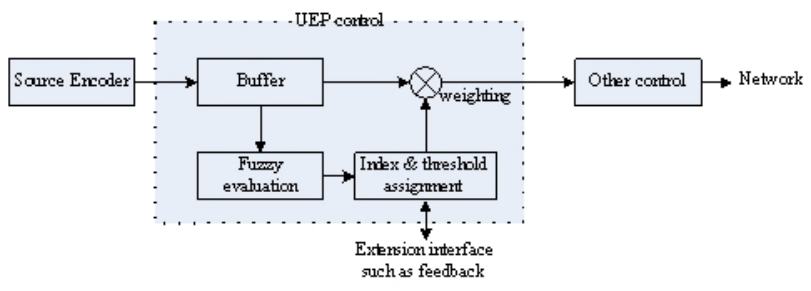

Fig 1. Diagram of UEP control based on fuzzy evaluation model

\section{SIMULATION RESULTS}

The test video sequence is 400 frames 'foreman' that was coded in QCIF format (176 x pixels/frame) at a temporal resolution of 30 (or 10) frames/s (fps) by the H.264 video coding standard in IPBP mode. Fig. 2 and Fig. 3 show the results by video evaluation model based on fuzzy judgment. It is encoded with a standard h.264 codec in IPBP mode. The frame rate of foreman sequence is $30 \mathrm{fps}$ in Fig. 2, while contrastive video frequency is 30fps in Fig. 3 . The quality values of $10 \mathrm{fps}$ segment have not been obviously affected by the varieties of PSNR (MSE), but the systematic evaluation results are not as good as the ones of $30 \mathrm{~Hz}$ due to increasing in both sampling interval and mobility which lead to worse fluency and wobble. Fig 4 shows the evaluation results for 'claire' sequence, which is a low motion video sequence with moderate contrast and noise and a uniform background. It is clear that the results are consistent with the judgments of subjective DSCQS (Double Stimulus Continuous Quality Scale). These results demonstrate the advantages the proposed objective video quality evaluation model based on fuzzy synthetic judgment, especially for the integrative video judgment.

Next, the sequence is protected according to the level provided by the proposed evaluation model, which is compared to the traditional proportional frame protection. Data partition is not introduced in the test due to serious error propagation, instead, frame copy technique is used to conceal the lost frames. It is because error propagation and concealment method affect the final video quality, so in this simulation, we just provide the affecting results of perceptual decision mode. For intensive study and experimentation on the relationship with error propagation, we will give in next research. The effectiveness of the two protection methods is measured by the peak signal-to-noise ratio (PSNR) and Video Quality Metric (VQM) software [8].

Fig 5 shows the comparison results of the Y-PSNR for the above two schemes with different frame loss rate. It can be seen from Fig 5 that our proposed scheme provides higher constructed video quality. Fig 6 shows the VQM testing results of the two schemes. It shows that our proposed schemes provide better perceived quality of the whole video sequence, even if the frame lose rate is close to $50 \%$.

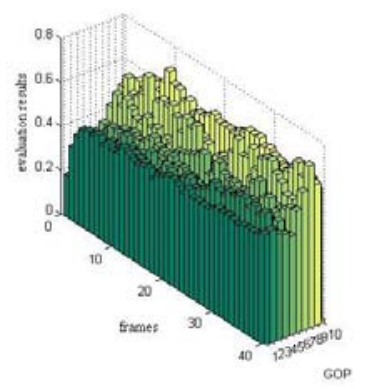

Fig 2. The evaluation result of foreman sequence of $30 \mathrm{~Hz}$

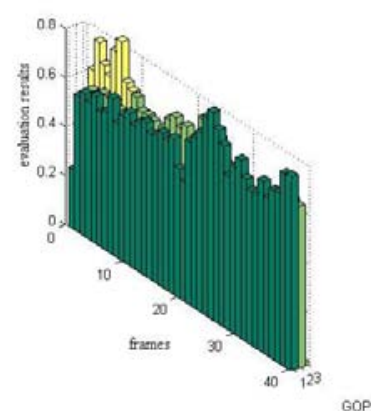

Fig. 3. The evaluation result of foreman sequence of $10 \mathrm{~Hz}$

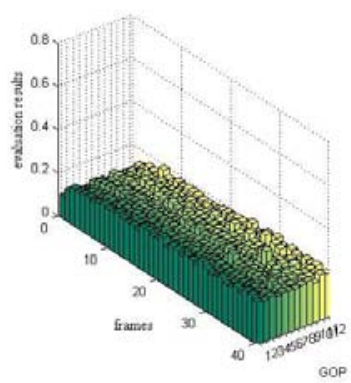

Fig. 4. The evaluation result of claire sequence of $30 \mathrm{~Hz}$

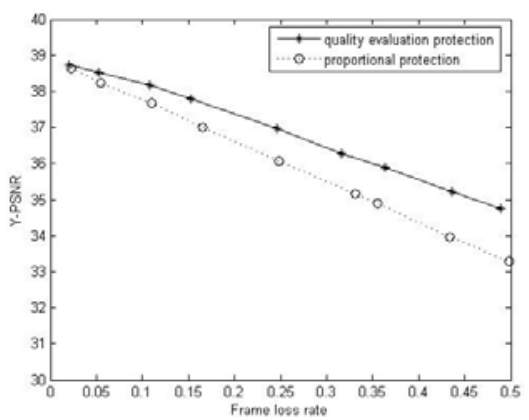

Fig 5. Comparison of PSNR for proposed UEP scheme vs. proportional UEP scheme 


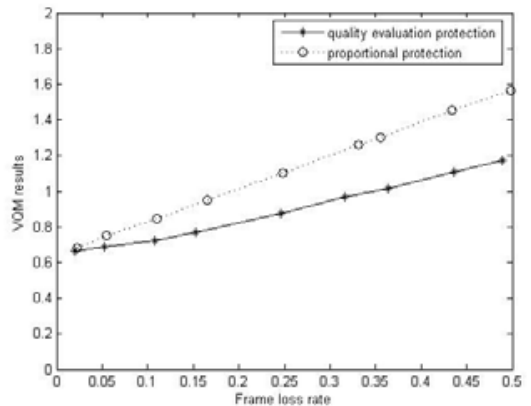

Fig 6. Comparison of VQM testing results for proposed UEP scheme vs. proportional UEP scheme

\section{CONCLUSION}

In this paper, we first develop an effective and systematic method for assessing the quality of compressed video by introducing multiple video affecting features into our model. Then, a novel unequal protection scheme is proposed based on the model. Both of the two methods have good compatibility with other models or algorithms. Simulation results demonstrate the advantages of the fuzzy objective video quality evaluation model, especially in comprehensive judgment of the whole video. The experimental results of the proposed unequal protection scheme based on the model also demonstrate the effectiveness for efficient transmission of compressed video, even in adverse environment with high frame loss rate.

\section{ACKNOWLEDGMENTS}

The authors would like to thank Prof. Haoshan Shi from Northwestern Polytechnical University for his help during this project, and the anonymous reviewers for their helpful comments.

\section{REFERENCES}

[1] Martini, M.G., Chiani, M., Proportional unequal error protection for MPEG-4 video transmission, in IEEE Conf. on Communications, vol 4, pp.1033 - 1037, June 2001.

[2] Z. Ahmad, S.T. Worrall, and A.H. Sadka, et al, A novel packetization scheme for MPEG-4 over 3G wireless systems, In IEE Conf. on $3 G$ Mobile Communication Technologies, pp. 302-306, October 2004.

[3] Wen Ji, Haoshan Shi,,et al., Objective Evaluation for Compressed Video Quality Based on Fuzzy Synthetic Judgment, In IEE Conf. on Fuzzy Systems and Knowledge Discover, pp.753-761, Sep. 2006.

[4] Jing Guo, Van Dyke-Lewis, et al., Gabor Difference Analysis of Digital Video Quality, IEEE Trans. Broadcasting, vol. 50, no. 3, pp: 302 - 311, 2004.

[5] Kuhmanch C., Schremmer C., Mpirical Evaluation of Layered Video Coding Schemes, in Conf. on Image Processing, vol 2, pp.1013 - 1016, Oct. 2001.

[6] Steiger O., Ebrahimi T.,et al., Evaluating Perceptually Prefitered Video, in IEE Conf. on Multimedia And Expo., pp.1290 - 1293, July 2005.

[7] Xiedong Cao., Fuzzy Information Processing and Application, Science Press,CN, 2003.

[8] Video Quality Studio, http://www.visumalchemia.com/ vqstudio/ 